\title{
Prevalence of Atherosclerosis in Psoriatic Patients Detected with Epiaortic Color Doppler Ultrasound and Computed Tomography Angiography
}

\author{
Annunziata Dattola ${ }^{1}$, Guglielmo Manenti ${ }^{2}$, Donatella Ferrari ${ }^{2}$, Laura Vollono ${ }^{1}$, \\ Salvatore Marsico ${ }^{2}$, Feliciana Lamacchia ${ }^{2}$, Maria Esposito ${ }^{1}$, Mattia Marchesano $^{1}$, \\ Arianna Zangrilli ${ }^{1}$, Roberto Floris ${ }^{2}$, Alessandro Giunta ${ }^{1}$, Luca Bianchi ${ }^{1}$
}

1 Dermatology Department, University of Rome “Tor Vergata”, Rome, Italy

2 Biomedicine and Prevention Department, UOC of Diagnostic Imaging, University of Rome “Tor Vergata”, Rome, Italy

Key words: psoriasis, color-doppler ultrasound, angio-CT, IMT, risk-factor

Citation: Dattola A, Manenti G, Ferrari D, et al. Prevalence of atherosclerosis in psoriatic patients detected with epiaortic color doppler ultrasound and computed tomograpgy angiography. Dermatol Pract Concept. 2022;12(1):e2022011.

DOI: https://doi.org/10.5826/dpc.1201a11

Accepted: May 6, 2021; Published: January 2022

Copyright: $\odot 2022$ Dattola et al. This is an open-access article distributed under the terms of the Creative Commons Attribution-NonCommercial License (BY-NC-4.0), https://creativecommons.org/licenses/by-nc/4.0/, which permits unrestricted noncommercial use, distribution, and reproduction in any medium, provided the original authors and source are credited.

Funding: None.

Competing interests: None.

Authorship: AD and GM have equally contributed to this article.

Corresponding author: Annunziata Dattola, MD, PhD. Dermatology Department, University of Rome “Tor Vergata”, Rome, Italy. E-mail: nancydattola@gmail.com

\footnotetext{
ABSTRACT Introduction: Psoriasis (PsO), a chronic inflammatory, multisystemic, and multifactorial disease can cause endothelial dysfunction, artery calcification, and atherosclerotic disease. A higher incidence of vascular occlusive events has been observed in psoriatic patients compared to healthy controls, and multiple studies confirm the association between moderate-severe $\mathrm{PsO}$ and atherosclerosis, coronary artery calcification, and higher cardiovascular risk.

Objective: We sought to analyze atherosclerotic disease prevalence in epiaortic vessels of psoriatic and non-psoriatic patients to understand if $\mathrm{PsO}$ could represent an independent risk factor predisposing to atherosclerotic disease.

Methods: We evaluated 47 psoriatic patients without cardiovascular risk factors with color Doppler ultrasound (CDUS). If atheromatous plaques were detected, a computed tomography angiography (CTA) was performed. We evaluated 47 non-psoriatic patients without cardiovascular risk factors with CDUS. Atherosclerosis prevalence in both groups were statistically analyzed. CDUS performance was compared to CTA.

Results: In the psoriatic group (mean age 50.9 years), 6 had atheromatous plaques and 12 had an intima-media thickness (IMT) $>1 \mathrm{~mm}$ (overall prevalence of atherosclerotic disease: $38.2 \%$ ). All plaques detected with CDUS were confirmed at CTA. In the control group (mean age 51.3 years),
} 
CDUS revealed atheromatous plaques in 4 patients and IMT $>1 \mathrm{~mm}$ in 4 ones (overall prevalence of $17 \%)$. The difference of atherosclerotic disease prevalence between the groups was statistically significant $(\mathrm{P}<0.05)$.

Conclusion: Our results highlight that $\mathrm{PsO}$ could be considered a predisposing factor for atherosclerotic disease development in epiaortic vessels, as it causes an increased IMT, that is also considered an independent cardiovascular risk factor.

\section{Introduction}

Psoriasis (PsO) is a chronic inflammatory, multisystemic, and multifactorial disease that affects about $3 \%$ of the world population [1]. The chronic systemic inflammatory process, that primarily affects the skin, also causes endothelial dysfunction, artery calcification, and atherosclerotic disease [2]. A higher incidence of vascular occlusive events has been observed in psoriatic patients compared to healthy controls, and multiple studies confirm the association between moderate-severe $\mathrm{PsO}$ and atherosclerosis, coronary artery calcification, and higher cardiovascular risk [3-8]. Young adults affected by severe PsO are 3 times more likely to face myocardial infarction than controls [9], and the risk of fatal myocardial infarction or stroke is even higher in patients hospitalized with PsO [10]. Moreover, a higher frequency of ischemic heart disease and cerebral and peripheral vascular disease has been observed in patients affected by moderate-severe disease compared with controls [11]. Thus, current opinion holds that PsO is an independent risk factor for cardiovascular disease [2,12,13].

Color Doppler ultrasonography (CDUS) of the carotid arteries is a first-level imaging technique performed in the diagnosis of carotid atherosclerotic disease. Ultrasonography (US) is the modality of choice for screening, diagnosis, and monitoring atheromatous disease because it is a fast, non-invasive, low-cost technique that evaluates the macroscopic appearance of atheromatous plaques as well as flow characteristics in the carotid artery. Although US is considered a useful tool in the assessment of the cardiovascular risk, there are few studies so far conducted regarding its use in patients affected by psoriasis vulgaris and psoriatic arthritis.

Computerized tomography angiography (CTA) is a very accurate technique for evaluating carotid stenosis, outperforming CDUS [14], but not as accurate in describing carotid plaques and assessing their vulnerability.

\section{Objectives}

The aim of this study is to evaluate the prevalence of atherosclerotic disease in epiaortic vessels of psoriatic patients without conventional cardiovascular risk factors, using CDUS and CTA, and to analyze the presence of any difference in prevalence with non-psoriatic patients, in order to understand if $\mathrm{PsO}$ could be considered as an independent risk factor for atherosclerotic disease.

\section{Methods}

This study was conduct in collaboration between the Departments of Dermatology and Diagnostic Imaging at the University of Rome Tor Vergata. The study was conducted from January 2017 to July 2017 at the Department of Dermatology. We enrolled 280 consecutive patients (males and females) with psoriasis vulgaris or arthropathic psoriasis. The patients underwent clinical and laboratory exams, including abdominal circumference measurements and arterial pressure. We determined if the patients were cigarette smokers and if had hypercholesterolemia, hypertriglyceridemia, and glycemia.

The inclusion criteria of the study were: male and female patients aged 35-85 years, with an abdominal circumference $<102 \mathrm{~cm}$ for males and $<88 \mathrm{~cm}$ for females, cholesterol plasmatic level $<200 \mathrm{mg} / \mathrm{dl}$, plasma glucose level $<126 \mathrm{mg} / \mathrm{dl}$ on 2 separate analyses, systolic pressure $<140 \mathrm{mmHg}$ and diastolic pressure $<90 \mathrm{mmHg}$, and non-smoker or $<5$ cigarettes/day smoker.

The exclusion criteria were: contraindications to iodinated contrast agent administration, pregnancy, regular use of alcohol (> 2 drinks/day), oncological history, family history of vascular disease, transient ischemic attack or stroke in the previous 5 years, and current or past use of intravenous drugs.

The final study group was composed of 47 psoriatic patients (patient group: 26 males and 21 females, aged 35-70 years), without conventional cardiovascular risk factors, and an equal number of non-psoriatic subjects, who had undergone epiaortic vessel CDUS at the Department of Diagnostic Imaging, recruited retrospectively between January 2017 and July 2017, (control group: 27 males and 20 females, aged 35-70 years). They underwent CDUS as a general exam, and from the medical interview carried out before the US examination it was determined they were in good health with no noteworthy diseases or underlying conditions, and without conventional cardiovascular risk factors.

Among the psoriatic patients, 37 were affected by psoriasis vulgaris and 10 by severe psoriatic arthritis, and all of 
them were being treated with systemic drugs including cyclosporine and/or methotrexate and anti-tumor necrosis factor $\alpha$ (TNF- $\alpha$ ) biologic drugs. All the subjects gave informed consent prior to their inclusion in the study.

All the subjects underwent epiaortic vessels CDUS. These evaluations were executed with a high-resolution US equipment (Philips iU22 Ultrasound System,), using a high-frequency linear probe $(9-3 \mathrm{MHz})$ and a carotid preset. The neck vessels studied were common carotid arteries, internal carotid arteries and external carotid arteries, and we evaluated both side regions of the neck for each subject for a total of 94 common carotid arteries, 94 internal carotid arteries, and 94 external carotid arteries in the psoriatic patients and an equal number of vessels in the non-psoriatic subjects. The vertebral arteries were examined in all participants, but the results obtained were not included in the study.

The US examinations were carried out by a radiologist with 5 years of experience, and the images were evaluated in consensus with a radiologist with 25 years of experience. The US exams were performed at first in B-mode with axial and longitudinal scans in order to identify arterial wall intima-media thickness (IMT) of common carotid arteries and the presence of common and internal carotid plaques.

The IMT measurement was performed automatically with dedicated software. The mean value between left and right sides was taken into consideration and an IMT $<1 \mathrm{~mm}$ was considered normal. The presence of a plaque was considered when a focal IMT was greater than $50 \%$ of the surrounding area [15]. Subsequently CDUS, and pulsed Doppler spectral analysis were performed: peak systolic velocity (PSV $\mathrm{cm} / \mathrm{s}$ ) and end diastolic velocity $(\mathrm{EDV} \mathrm{cm} / \mathrm{s})$ values were analyzed to determine the percentage of stenosis and plaque hemodynamics.

When at least 1 carotid plaque or an increased IMT of the common carotid on a side was detected with US examination, the patient was considered positive for atherosclerotic disease. If both carotids had an IMT $>1 \mathrm{~mm}$, the average thickness of the 2 was taken into consideration.

In the psoriatic patient group, if CDUS examination detected atherosclerotic plaque, the patient had to go simultaneous CTA of the extracranial vessels in order to confirm its presence, better define plaque features, determine the percentage of stenosis, and search for the presence of additional plaques in other locations. Psoriatic patients who at CDUS presented an IMT $>1 \mathrm{~mm}$ were asked to follow-up after 12 months with another CDUS to check for vascular disease. The psoriatic patients who did not present any atherosclerotic pathology at CDUS were asked to follow-up with regular dermatological visits.

Subjects of the control group underwent epiaortic vessel CDUS. If a plaque revealed severe stenosis (> 70\%) or if signs of surface ulceration were detected, they underwent a CTA to ensure adequate therapeutic diagnostic planning of the incidental finding, according to the current clinical practice. The CTA was performed using a Lightspeed VCT 64-slice scanner (General Electric), with a pre-contrast phase and an arterial phase with bolus tracking, with a non-ionic iodinated contrast agent injection $(350 \mathrm{mg} / \mathrm{ml})$ with a flow rate of $3.5 \mathrm{ml} / \mathrm{s}$, followed by a bolus of $20 \mathrm{ml}$ of saline flush at the same speed. The images were also analyzed in $3 \mathrm{D}$, with multiplanar reconstruction and volume rendering and interpreted in consensus with the same two radiologists. When a plaque was detected, the carotid stenosis was quantified by the NASCET method: (distal diameter of the internal carotid - residual diameter / distal diameter of the internal carotid) $\times 100$ [16]. The radiological features of the detected plaques analyzed with CDUS and CTA were the percentage of stenosis, structure, and surface.

Continuous variables, normally distributed (patient age, IMT thickness) were presented as the mean \pm standard deviation (SD) and categorical variables were presented as counts (percentage). Fisher exact test was used to compare differences in atherosclerotic disease prevalence between psoriatic patients and healthy subjects. Any $\mathrm{P}$ value $<0.05$ was considered statistically significant.

\section{Results}

The mean (SD) age of the psoriatic group was $50.9 \pm 8$ years (range 35-70 years), and of the control group $51.3 \pm 8$ years (range 35-70 years). In the psoriatic patients an IMT $>1 \mathrm{~mm}$ without carotid plaques (Figure 1) was found in 12 patients $(25.5 \%)$, with a mean value of $1.4 \mathrm{~mm}$ (range 1.1-1.8 $\mathrm{mm}$ ). Atheromatous plaques were found in 6 patients, mean age $57.5 \pm 4.6$ years (range 50-64 years), with a prevalence of $12.7 \%$. In 4 patients, an increase of IMT coexisted, thus the

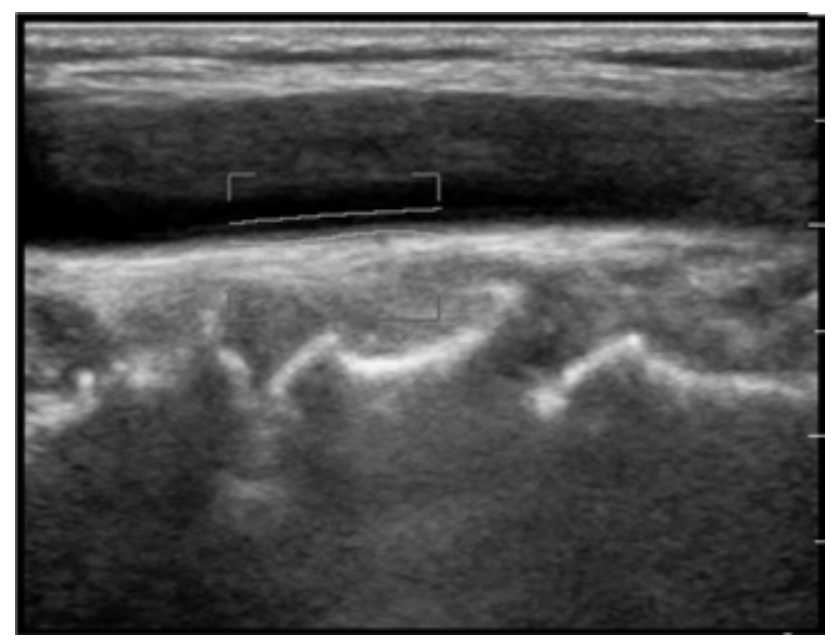

Figure 1. Longitudinal US of the right common carotid artery of a 51-year-old female psoriatic patient with an IMT $>1 \mathrm{~mm}$ without plaque. IMT $=$ intima-media thickness; US = ultrasonography. 
increase of IMT was detected in a total of 16 patients with a prevalence of $34 \%$. A total number of 18 patients showed carotid atherosclerotic disease with an overall prevalence of $38.2 \%$.

Plaques were found in 6 patients and were localized in carotid arteries bilaterally in each patient, with a total number of 12 plaques. Among these, US revealed 2 plaques located in internal carotid artery (ICA), 4 plaques located in carotid bifurcations, and 6 plaques located in carotid bifurcations extended to the ipsilateral ICA. Moreover, the structure of the plaques was hypoechoic in 4 cases $(33 \%)$ and fibrocalcific in
8 cases $(67 \%)$. The plaque surface was smooth $(83.4 \%)$ or irregular $(16.6 \%)$, no ulceration signs were detected at US examination. In these 12 cases the plaques revealed mild stenosis, with a percentage of stenosis variable at $10-30 \%$. Pulsed Doppler spectral analysis consistently showed a PSV $<125$ $\mathrm{cm} / \mathrm{s}$ with an EDV $<40 \mathrm{~cm} / \mathrm{s}$ in the ICA, demonstrating that the stenosis was not hemodynamically significant (Figure 2).

All plaques detected with CDUS analysis were confirmed with CTA examination (Figure 3). At the final CTA, 4 plaques were in a left bifurcation and ICA $(33.3 \%), 3$ plaques were in a right bifurcation and ICA $(25 \%), 2$ plaques were in a

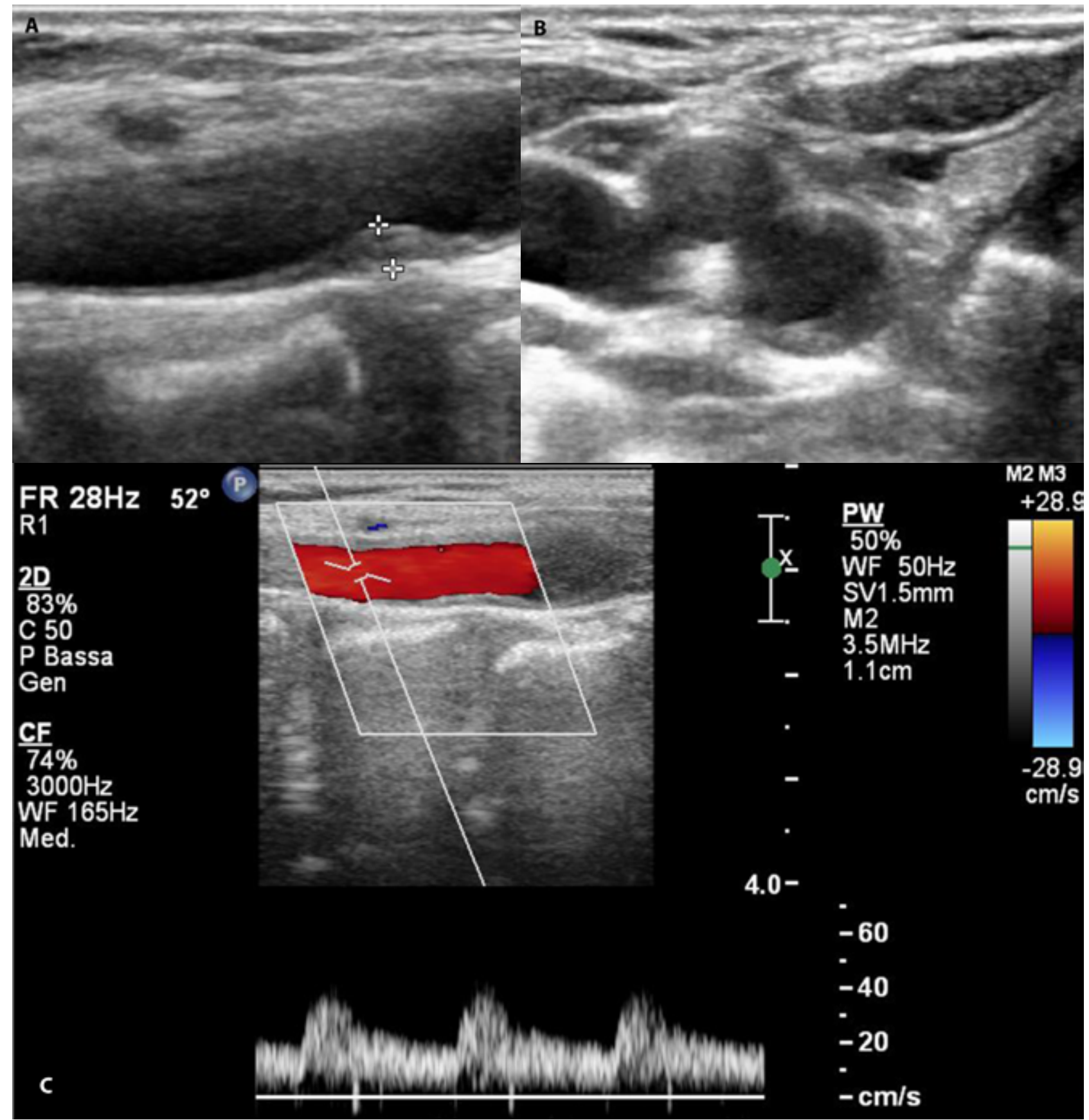

Figure 2. (A) Longitudinal ultrasonography of a hypoechoic plaque in the right internal carotid artery of a 50-year-old male psoriatic patient with a smooth surface that produced stenosis of about $25 \%$. (B) Axial ultrasonography of the same plaque. (C) longitudinal color Doppler ultrasonography and pulsed Doppler spectral analysis of this plaque showing a peak systolic velocity of $40 \mathrm{~cm} / \mathrm{s}$, not hemodynamically significant. 
right bifurcation (16.6\%), 1 in a left bifurcation $(8.3 \%), 1$ in a right ICA $(8.3 \%)$ and 1 in a left ICA $(8.3 \%) ; 8$ plaques were fibrocalcific $(67 \%), 2$ hypoechoic plaques which were previously documented at US showed a wide fibrous cap (16.5\%), and 2 were lipid-rich plaques (16.5\%) (Table 1$)$. All of them produced a stenosis $<30 \%$ and none of them showed features of vulnerable plaques following both CDUS and CTA examinations. Our results showed good agreement between the two imaging techniques, with a concordance rate of $92 \%$ between CDUS and CTA regarding plaque localization, $83 \%$ regarding plaque structure, and $100 \%$ regarding both percentage of stenosis and plaque surface morphology.

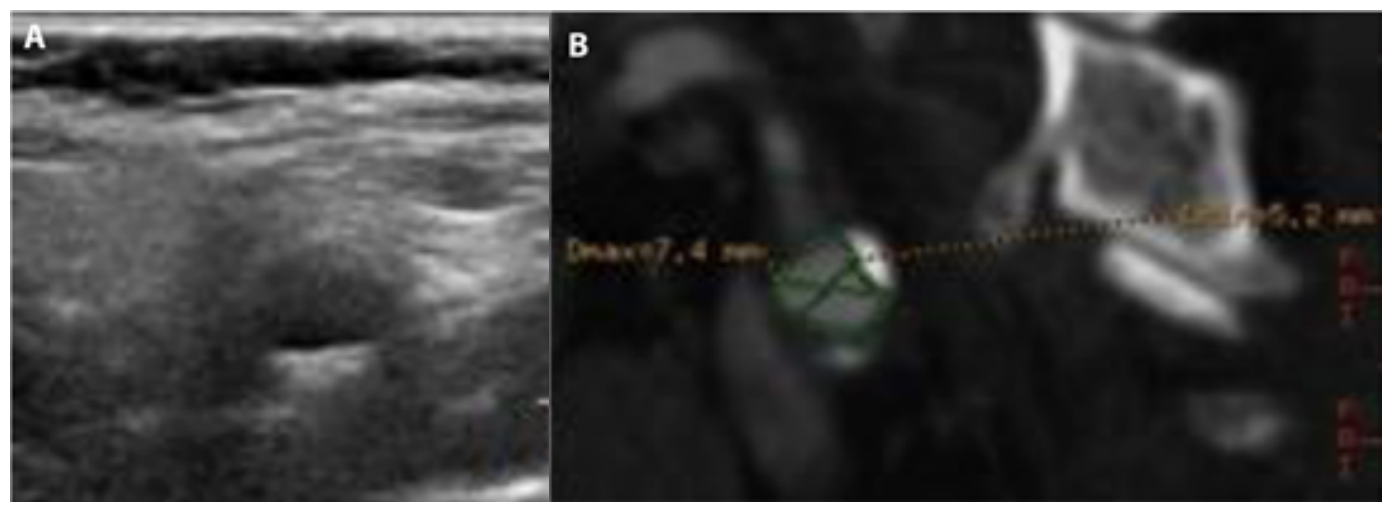

Figure 3. (A) Axial ultrasound of a fibrocalcific plaque in left internal carotid artery of a 58 -year-old male psoriatic patient, with a smooth surface that produced stenosis of about $20 \%$. (B) Oblique reconstruction of computed tomography angiography of the plaque that produced a stenosis of about $20 \%$.

Table 1. Epidemiological Characteristics of Psoriatic Patients with Plaques $(n=6)$ and Features of These Plaques $(\mathrm{n}=12)$ With CDUS and CTA Examinations*

\begin{tabular}{|c|c|c|c|c|c|}
\hline Psoriatic Patient & Technique & Location & Stenosis & Structure & Surface \\
\hline \multirow{4}{*}{ Male, 60 years } & CDUS & Right bifurcation + ICA & $30 \%$ & Fibrocalcific & Irregular \\
\hline & CTA & Right bifurcation + ICA & $30 \%$ & Fibrocalcific & Irregular \\
\hline & CDUS & Left bifurcation + ICA & $30 \%$ & Fibrocalcific & Irregular \\
\hline & CTA & Left bifurcation + ICA & $30 \%$ & Fibrocalcific & Irregular \\
\hline \multirow{4}{*}{ Male, 50 years } & CDUS & Right ICA & $25 \%$ & Lipid-rich & Smooth \\
\hline & CTA & Right ICA & $25 \%$ & Lipid-rich & Smooth \\
\hline & CDUS & Left bifurcation + ICA & $20 \%$ & Fibrocalcific & Smooth \\
\hline & CTA & Left bifurcation + ICA & $20 \%$ & Fibrocalcific & Smooth \\
\hline \multirow{4}{*}{ Male, 58 years } & CDUS & Right bifurcation & $15 \%$ & Fibrocalcific & Smooth \\
\hline & CTA & Right bifurcation & $15 \%$ & Fibrocalcific & Smooth \\
\hline & CDUS & Left ICA & $20 \%$ & Fibrocalcific & Smooth \\
\hline & CTA & Left ICA & $20 \%$ & Fibrocalcific & Smooth \\
\hline \multirow{4}{*}{ Female, 64 years } & CDUS & Right bifurcation + ICA & $30 \%$ & Fibrocalcific & Smooth \\
\hline & CTA & Right bifurcation + ICA & $30 \%$ & Fibrocalcific & Smooth \\
\hline & CDUS & Left bifurcation & $20 \%$ & Lipid-rich & Smooth \\
\hline & CTA & Left bifurcation + ICA & $20 \%$ & Lipid-rich & Smooth \\
\hline \multirow{4}{*}{ Female, 57 years } & CDUS & Right bifurcation & $20 \%$ & Lipid-rich & Irregular \\
\hline & CTA & Right bifurcation & $20 \%$ & Fibrous + lipid core & Irregular \\
\hline & CDUS & Left bifurcation + ICA & $10 \%$ & Fibrocalcific & Smooth \\
\hline & CTA & Left bifurcation + ICA & $10 \%$ & Fibrocalcific & Smooth \\
\hline \multirow{4}{*}{ Female, 56 years } & CDUS & Right bifurcation + ICA & $15 \%$ & Fibrocalcific & Smooth \\
\hline & CTA & Right bifurcation + ICA & $15 \%$ & Fibrocalcific & Smooth \\
\hline & CDUS & Left bifurcation & $20 \%$ & Lipid-rich & Smooth \\
\hline & CTA & Left bifurcation & $20 \%$ & Fibrous + lipid core & Smooth \\
\hline
\end{tabular}

* CDUS errors compared to CTA are highlighted in gray.

CDUS = color Doppler ultrasonography; CTA = computed tomography angiography; ICA = internal carotid artery . 
Moreover, CTA did not reveal the presence of additional plaques along the carotid axes or along vertebral arteries.

Regarding subjects of the control group, US examination revealed IMT > $1 \mathrm{~mm}$ without carotid atheromatous plaques in 4 people $(8.5 \%)$ bilateral or unilateral, with a mean value of $1.2 \mathrm{~mm}$ (range 1.1-1.5). Atheromatous plaques were detected in 4 subjects, mean age $62.5 \pm 2.6$ years (range 59-64), with a prevalence of $8.5 \%$. Plaques were unilateral in 2 cases and bilateral in 2 cases, with a total number of 6 plaques. The structure of these plaques was hypoechoic in 2 cases $(33.3 \%)$, and fibrocalcific in 4 cases $(66.7 \%)$, with a variable percentage of stenosis at $10-20 \%$, and none of them were hemodynamically significant at color Doppler analysis. Other plaque features of the control group subjects are explained in Table 2. In 2 cases an increased IMT coexisted, thus an increased IMT was detected in a total of 6 subjects with a prevalence of $12.7 \%$. A total number of 8 cases showed carotid atherosclerotic disease with an overall prevalence of $17 \%$. The Fisher exact test statistic value was 0.0368 , and this result highlights the presence of a statistically significant difference $(\mathrm{P}<0.05)$ between the prevalence of carotid atherosclerotic disease (increase of IMT and/or atheromatous plaques) in patients without cardiovascular risk factors affected by $\mathrm{PsO}$ and those without cardiovascular risk factors and without PsO.

\section{Conclusions}

$\mathrm{PsO}$ is a chronic systemic inflammatory disease. With the term "psoriasis march," Boehncke et al described the process that starts from genetic and environmental predisposing factors and leads to a dysregulation of the immune system that gives rise to a chronic systemic inflammatory state that also causes endothelial dysfunction, atherosclerosis, and coronary events.
Innate and adaptive immune systems are likewise considered responsible for pathological changes in both epidermis and vascular walls [2].

A recent meta-analysis of 4 genome-wide association studies selected a broad number of single nucleotide polymorphisms (SNPs) that showed a significant association with cardiovascular disease, hypertension, body mass index, hyperlipidemia, or type II diabetes. They then investigated which one of these polymorphisms was also associated with $\mathrm{PsO}$ [17] and identified 8 main SNPs codifying for a key protein in the process of lymphocyte differentiation, thrombogenesis, and in the induction of VCAM1 and E-selectin on the surface of endothelial cells under the stimulation of TNF- $\alpha$. This might explain its dual role in establishing a susceptibility to multiple immune-mediated and cardiovascular diseases. Subcutaneous tissue is able to produce proinflammatory cytokines and C-reactive protein under the influence of mediators such as TNF- $\alpha$ [18].

Modifications in the adipocyte metabolic profile, including alterations of adiponectin, leptin and resistin, are considered responsible in starting or maintaining the inflammatory process [19]. In particular, high blood levels of resistin seem to be related to a proinflammatory systemic state, insulin-resistance, and atherosclerosis [2]. Metabolic syndrome is more frequent in psoriatic patients than in the general population [12], and its prevalence directly correlates to PsO duration and severity [20]. Of note, the abovementioned effects are particularly relevant when dealing with central or visceral obesity [21]. A direct correlation between severity of $\mathrm{PsO}$, central obesity and vascular inflammation has been reported, with concurrent reduction of these parameters after 12 months of systemic or biologic therapy [22].

Table 2. Epidemiological Characteristics of Non-Psoriatic Patients $(n=8)$ with Atheromatous Pathology (IMT >1 mm and/or plaques) and Features of the Plaques $\left(n^{\circ}=6\right)$ Detected With CDUS

\begin{tabular}{|c|c|c|c|c|c|}
\hline Non-Psoriatic Subjects & Location & Pathology & Stenosis & Structure & Morphology \\
\hline \multirow[t]{2}{*}{ Male, 64 years } & $\begin{array}{l}\text { Right bifurcation } \\
\text { Left bifurcation }\end{array}$ & $\begin{array}{l}\text { Plaque } \\
\text { Plaque }\end{array}$ & $\begin{array}{l}15 \% \\
20 \% \\
\end{array}$ & $\begin{array}{c}\text { Lipid-rich } \\
\text { Fibrocalcific }\end{array}$ & $\begin{array}{l}\text { Smooth } \\
\text { Smooth }\end{array}$ \\
\hline & Bilateral & IMT 1.2 & - & - & - \\
\hline Male, 65 years & Right bifurcation + ICA & Plaque & $20 \%$ & Fibrocalcific & Smooth \\
\hline Male, 59 years & Left bifurcation + ICA & Plaque & $10 \%$ & Lipid-rich & Smooth \\
\hline \multirow[t]{2}{*}{ Female, 62 years } & $\begin{array}{l}\text { Left bifurcation } \\
\text { Right bifurcation + ICA }\end{array}$ & $\begin{array}{l}\text { Plaque } \\
\text { Plaque }\end{array}$ & $\begin{array}{l}15 \% \\
15 \% \\
\end{array}$ & $\begin{array}{l}\text { Fibrocalcific } \\
\text { Fibrocalcific }\end{array}$ & $\begin{array}{l}\text { Smooth } \\
\text { Smooth }\end{array}$ \\
\hline & Bilateral & IMT 1.2 & - & - & - \\
\hline Female, 61 years & Right & IMT 1.2 & - & - & - \\
\hline Male, 68 years & Bilateral & IMT 1.3 & - & - & - \\
\hline Male, 61 years & Left & IMT 1.1 & - & - & - \\
\hline Female, 62 years & Bilateral & IMT 1.1 & - & - & - \\
\hline
\end{tabular}

CDUS = color Doppler ultrasonography; ICA = internal carotid artery; IMT = intima-media thickness. 
Recently, sonoelastography has been proposed as a tool in monitoring the response to treatment in psoriatic patients, as it evaluates hypodermal adipose tissue inflammation underneath lesioned plaques at baseline and during systemic treatment [23].

Twenty-five years ago, McDonald and Calabresi performed the first study showing that patients with $\mathrm{PsO}$ had more vascular occlusive events than patients without $\mathrm{PsO}$ [3]. Subsequently, multiple studies confirmed the association between moderate-severe $\mathrm{PsO}$ and atherosclerosis, coronary artery calcification, higher cardiovascular risk, myocardial infarction, stroke and peripheral vascular disease [4-11].It is hypothesized that this association might be due to an overrepresentation in the psoriatic population with a Framingham Risk Score (age, hypertension, obesity, smoking, diabetes, hypercholesterolemia, hyperlipidemia, and familial history) [12]. In addition, many traditional systemic therapies for PsO seem to affect cardiovascular risk, increasing cardiovascular risk factors such as hyperlipidemia, hypertension, and hyperhomocysteinemia. Nevertheless, recent studies identified $\mathrm{PsO}$ as an independent risk factor for cardiovascular disease [9,10,11,12,13,20].

An increased prevalence and severity of coronary artery calcification and atherosclerosis (measured by cardiac computed tomography, CTA, or coronary angiography) has been reported in psoriatic patients compared to healthy controls [24-30]. A lower coronary flow reserve has been observed in young subjects, otherwise healthy patients with severe $\mathrm{PsO}$ compared to controls, suggesting that early impairment of coronary microvascular function is independent of conventional cardiovascular risk factors.

Our study reveals abnormalities in the vessel walls of carotid arteries in a great percentage of psoriatic patients who did not have any cardiovascular risk factors. In particular, we highlighted a higher prevalence of carotid atherosclerotic disease (increased IMT and/or atheromatous plaques) in patients affected by $\mathrm{PsO}$ than in healthy subjects regardless of cardiovascular risk factors. It was detected in an increased IMT, which represents a predisposing risk factor for stroke events [31]. Analysis of the data also showed a higher mean age in the control group affected by atheromatous plaques than that of psoriatic patients affected by atheromatous plaques. In fact, in the control group plaques were identified only in subjects with advanced age (subjects older than 55 years, the average age of the group); on the contrary, in psoriatic group, plaques were also identified in younger patients. This also led us to the conclusion that $\mathrm{PsO}$ can be considered an independent risk factor in the development of atheromatous pathology. Our results highlighted a good agreement between CDUS and CTA in atheromatous plaque detection and plaque analysis, showing a $92 \%$ concordance rate between CDUS and CTA regarding plaque localization, $83 \%$ regarding plaque structure, and $100 \%$ regarding both percentage of stenosis and plaque surface morphology.

Ultrasound errors were made only in determining the plaque composition in 2 cases ( 2 hypoechoic plaques previously documented at US showed a wide fibrous cap at CTA), and in determining the complete plaque extension along the vessel in 1 case (a plaque that at US examination seemed localized only in the carotid bifurcation and at CTA appeared extended to the ipsilateral ICA). In spite of being a fast, noninvasive, well tolerated, and low-cost imaging method, US is an operator-dependent examination that requires a good understanding of Doppler physics and hemodynamic physiology. A more precise evaluation of the percentage of stenosis, of the composition and of the morphology of the plaque is made with CTA, but it uses ionizing radiation and potentially allergenic and nephrotoxic contrast agents. In fact, CTA has been proved to be an accurate modality for detection of severe carotid artery disease and, especially, for detection of occlusions [32]. This means that CDUS should not be replaced as the first-line investigation for epiaortic vessels.

Although our results are based on a small cohort of patients, we concluded that $\mathrm{PsO}$ could be considered a risk factor for the development of atheromatous pathology in epiaortic vessels, because primarily it causes a thickening of the IMT. Therefore, this study contributes to support the hypothesis that PsO should be considered as an independent cardiovascular risk factor.

The American Society of Echocardiography more recently recommended the cut-off value for IMT at $\geq 1.5 \mathrm{~mm}$, but set IMT above $1 \mathrm{~mm}$ [15]. The main limitation of this study is the small cohort of subjects included and, in this regard, further studies with larger cohorts, carried on in close cooperation with different medical specialists, finalized to a correct selection of patients, are necessary to confirm and improve our results. This could be relevant in order to aid in the clinical management of psoriatic patients and offer them better diagnostic/therapeutic pathways to prevent cardiovascular disease.

We propose that epiaortic CDUS as the most suitable method in assessing cardiovascular risk and in the early identification of minor changes and atheromatous plaques of epiaortic vessels of psoriatic patients.

\section{References}

1. Dattola A, Cannizzaro MV, Mazzeo M, Bianchi L. Certolizumab Pegol in the Treatment of Psoriasis and Psoriatic Arthritis: Preliminary Real-Life Data. Dermatol Ther (Heidelb). 2017;7(4):485492. DOI: 10.1007/s13555-017-0208-z. PMID: 29139035; PMCID: PMC5698207.

2. Boehncke S, Thaci D, Beschmann $H$, et al. Psoriasis patients show signs of insulin resistance. Br J Dermatol. 2007;157(6):1249-1251. DOI: 10.1111/j.1365-2133.2007.08190.x. PMID: 17916217.

3. McDonald CJ, Calabresi P. Psoriasis and occlusive vascular disease. Br J Dermatol. 1978;99(5):469-745. DOI: 10.1111/j.13652133.1978.tb02012.x. PMID: 708620 
4. Abuabara K, Azfar RS, Shin DB, Neimann AL, Troxel AB, Gelfand JM. Cause-specific mortality in patients with severe psoriasis: a population-based cohort study in the U.K. Br J Dermatol. 2010;163(3):586-592. DOI: 10.1111/j.1365-2133.2010.09941.x. PMID: 20633008; PMCID: PMC2966545.

5. Ahlehoff O. Gislason GH, Jørgensen $\mathrm{CH}$, et al. Psoriasis and risk of atrial fibrillation and ischaemic stroke: a Danish Nationwide Cohort Study. Br J Dermatol. 2007;157(6):1249-5121. DOI: 10.1111/j.1365-2133.2007.08190.x. PMID: 17916217.

6. Mehta NN, Yu Y, Pinnelas R, et al. Attributable risk estimate of severe psoriasis on major cardiovascular events. Am J Med. 2011;124(8):775.e1-6. DOI: 10.1016/j.amjmed.2011.03.028. PMID: 21787906; PMCID: PMC3146037.

7. Ludwig RJ, Herzog C, Rostock A, et al. Psoriasis: a possible risk factor for development of coronary artery calcification. Br J Dermatol. 2007;156(2):271-276. DOI: 10.1111/j.13652133.2006.07562.x. PMID: 17223866.

8. Brauchli YB, Jick SS, Miret M, Meier CR. Psoriasis and risk of incident myocardial infarction, stroke or transient ischaemic attack: an inception cohort study with a nested case-control analysis. Br J Dermatol. 2009;160(5):1048-1056. DOI: 10.1111/j.13652133.2008.09020.x. PMID: 19210501.

9. Gelfand JM, Neimann AL, Shin DB, Wang X, Margolis DJ, Troxel AB. Risk of myocardial infarction in patients with psoriasis. JAMA. 2006; 296(14):1735-1741. DOI: 10.1001/ jama.296.14.1735. PMID: 17032986.

10. Mallbris L, Akre O, Granath F, et al. Increased risk for cardiovascular mortality in psoriasis inpatients but not in outpatients. Eur J Epidemiol. 2004;19(3):225-230. DOI: 10.1023/b:ejep.0000020447.59150.f9. PMID: 15117115.

11. Prodanovich S, Kirsner RS, Kravetz JD, Ma F, Martinez L, Federman DG. Association of psoriasis with coronary artery, cerebrovascular, and peripheral vascular diseases and mortality. Arch Dermatol. 2009 Jun;145(6):700-703. DOI: 10.1001/archdermatol.2009.94. PMID: 19528427.

12. Gisondi P, Tessari G, Conti A, et al. Prevalence of metabolic syndrome in patients with psoriasis: a hospital-based case-control study. Br J Dermatol. 2007;157(1):68-73. DOI: 10.1111/j.13652133.2007.07986.x. Epub 2007 Jun 6. PMID: 17553036.

13. Mehta NN, Azfar RS, Shin DB, Neimann AL, Troxel AB, Gelfand JM. Patients with severe psoriasis are at increased risk of cardiovascular mortality: cohort study using the General Practice Research Database. Eur Heart J. 2010;31(8):1000-1006. DOI: 10.1093/ eurheartj/ehp567. PMID: 20037179; PMCID: PMC2894736.

14. Anzidei M, Napoli A, Zaccagna F, et al. Diagnostic Accuracy of Colour Doppler Ultrasonography, CT Angiography and BloodPool-Enhanced MR Angiography in Assessing Carotid Stenosis: A Comparative Study With DSA in 170 Patients. Radiol Med. 2012 Feb;117(1):54-71. DOI: 10.1007/s11547-011-0651-3. PMID: 21424318.

15. Johri AM, Nambi V, Naqvi TZ, et al. Recommendations for the Assessment of Carotid Arterial Plaque by Ultrasound for the Characterization of Atherosclerosis and Evaluation of Cardiovascular Risk: From the American Society of Echocardiography. J Am Soc Echocardiogr. 2020;33(8):917-933. DOI: 10.1016/j. echo.2020.04.021. PMID: 32600741.

16. Saba L, Mallarini GA. A comparison between NASCET and ECST methods in the study of carotids: evaluation using Multi-Detector-Row CT angiography. Eur J Radiol. 2010;76(1):42-47. DOI: 10.1016/j.ejrad.2009.04.064. PMID: 19464837.
17. Ikäheimo I, Tiilikainen A, Karvonen J, Silvennoinen-Kassinen S. HLA risk haplotype Cw6,DR7,DQA1*0201 and HLA-Cw6 with reference to the clinical picture of psoriasis vulgaris. Arch Dermatol Res. 1996;288(7):363-365. DOI: 10.1007/BF02507104. PMID: 8818183.

18. Meijer K, de Vries M, Al-Lahham S, et al. Human primary adipocytes exhibit immune cell function: adipocytes prime inflammation independent of macrophages. PLoS One. 2011;6(3):e17154. DOI: 10.1371/journal.pone.0017154. PMID: 21448265; PMCID: PMC3063154.

19. Puig L. Obesity and psoriasis: body weight and body mass index influence the response to biological treatment. J Eur Acad Dermatol Venereol. 2011;25(9):1007-1011. DOI: 10.1111/j.14683083.2011.04065.x. PMID: 21492252.

20. Gisondi P, Fostini AC, Fossà I, Girolomoni G, Targher G. Psoriasis and the metabolic syndrome. Clin Dermatol. 2018;36(1):21-28. DOI: 10.1016/j.clindermatol.2017.09.005. PMID: 29241748.

21. Guidolin L, Borin M, Fontana E, Caroppo F, Piaserico S, Fortina AB. Central Obesity in Children with Psoriasis. Acta Derm Venereol. 2018;98(2):282-283. DOI: 10.2340/00015555-2816. PMID: 29048098.

22. Rivers JP, Powell-Wiley TM, Dey AK, et al. Visceral Adiposity in Psoriasis is Associated With Vascular Inflammation by $18 \mathrm{~F}-\mathrm{Flu}-$ orodeoxyglucose Positron-Emission Tomography/Computed Tomography Beyond Cardiometabolic Disease Risk Factors in an Observational Cohort Study. JACC Cardiovasc Imaging. 2018;11(2 Pt 2):349-357. DOI: 10.1016/j.jcmg.2017.08.014. PMID: 29055628; PMCID: PMC5803350.

23. Dattola A, Altobelli S, Marsico S, et al. Hypodermal Adipose Tissue Sonoelastography for Monitoring Treatment Response in Patients with Plaque Psoriasis. Photomed Laser Surg. 2017;35(9):484491. DOI: 10.1089/pho.2016.4261. PMID: 28445107.

24. Mansouri B, Kivelevitch D, Natarajan B, et al. Comparison of Coronary Artery Calcium Scores Between Patients With Psoriasis and Type 2 Diabetes. JAMA Dermatol. 2016 Nov 1;152(11):1244-1253. DOI: 10.1001/jamadermatol.2016.2907. PMID: 27556410.

25. Staniak HL, Bittencourt MS, De Souza Santos I, et al. Association between psoriasis and coronary calcium score. Atherosclerosis. 2014;237(2):847-852. DOI: $10.1016 / \mathrm{j}$. atherosclerosis.2014.11.004. PMID: 25463132.

26. Bissonnette R, Cademartiti F, Maffei E, Tardif JC. Increase in coronary atherosclerosis severity and the prevalence of coronary artery mixed plaques in patients with psoriasis. Br J Dermatol. 2017;176(3):800-802. DOI: 10.1111/bjd.14797. PMID: 27291937.

27. Hjuler KF, Böttcher M, Vestergaard C, et al. Increased Prevalence of Coronary Artery Disease in Severe Psoriasis and Severe Atopic Dermatitis. Am J Med. 2015;128(12):1325-34.e2. DOI: 10.1016/j.amjmed.2015.05.041. PMID: 26093174.

28. Picard D, Bénichou J, Sin C, et al. Increased prevalence of psoriasis in patients with coronary artery disease: results from a case-control study. Br J Dermatol. 2014;171(3):580-587. DOI: 10.1111/ bjd.13155. PMID: 24904002.

29. Zeb I, Budoff M. Coronary artery calcium screening: does it perform better than other cardiovascular risk stratification tools? Int J Mol Sci. 2015;16(3):6606-6620. DOI: 10.3390/ijms16036606. PMID: 25807266; PMCID: PMC4394551.

30. Eckert J, Schmidt M, Magedanz A, Voigtländer T, Schmermund A. Coronary CT angiography in managing atherosclerosis. Int $\mathrm{J} \mathrm{Mol}$ 
Sci. 2015;16(2):3740-56. DOI: 10.3390/ijms16023740. PMID: 25671814 ; PMCID: PMC4346923.

31. Ebrahim S, Papacosta O, Whincup P, et al. Carotid plaque, intima media thickness, cardiovascular risk factors, and prevalent cardiovascular disease in men and women: the British Regional Heart Study. Stroke. 1999;30(4):841-850. DOI: 10.1161/01. str.30.4.841. PMID: 10187889.
32. Koelemay MJ, Nederkoorn PJ, Reitsma JB, Majoie CB. Systematic review of computed tomographic angiography for assessment of carotid artery disease. Stroke. 2004;35(10):2306-2312. DOI: 10.1161/01.STR.0000141426.63959.cc. PMID: 15345798. 